\title{
Delone Sets with Finite Local Complexity: Linear Repetitivity Versus Positivity of Weights
}

\author{
Adnene Besbes • Michael Boshernitzan • \\ Daniel Lenz
}

Received: 17 February 2012 / Revised: 29 May 2012 / Accepted: 27 August 2012 /

Published online: 5 September 2012

(C) Springer Science+Business Media, LLC 2012

\begin{abstract}
We consider Delone sets with finite local complexity. We characterize the validity of a subadditive ergodic theorem by uniform positivity of certain weights. The latter can be considered to be an averaged version of linear repetitivity. In this context, we show that linear repetitivity is equivalent to positivity of weights combined with a certain balancedness of the shape of return patterns.
\end{abstract}

Keywords Delone sets $\cdot$ Linear repetitivity $\cdot$ Subadditive ergodic theorem

\section{Introduction}

Aperiodic point sets with long range order have attracted considerable attention in recent years (see, e.g., the monographs and conference proceedings [1, 23, 24, 28]). On the one hand, this is due to the actual discovery of physical substances, later called quasicrystals, exhibiting such features [11,27]. On the other hand, this is due to intrinsic mathematical interest in describing the very border between crystallinity and aperiodicity. While there is no axiomatic framework for aperiodic order yet, various types of order conditions in terms of local complexity functions have

\footnotetext{
A. Besbes $(\bowtie)$

Université de Tunis El Manar, I.P.E.I de Bizerte, BP 64-7021, Zarzouna (Bizerte), Tunisia e-mail: abesbes@math.jussieu.fr

M. Boshernitzan

Rice University, Houston, TX 77251, USA

e-mail: michael@rice.edu

D. Lenz

Mathematisches Institut, Friedrich-Schiller Universität Jena, Ernst-Abbé Platz 2, 07743 Jena, Germany

e-mail: daniel.lenz@uni-jena.de
} 
been studied [12-14]. Here, we are concerned with linear repetitivity. This condition is given by a linear bound on the growth rate of the repetitivity function. It has been brought forward by Lagarias-Pleasants in [13] as a characterization of perfectly ordered quasicrystals. In fact, as shown in [14], among the aperiodic repetitive Delone sets, linearly repetitive ones have the slowest possible growth rate of their repetitivity function.

Let us also note that independent of the work of Lagarias-Pleasants, the analogue notion for subshifts has received a thorough study in works of Durand [6, 7].

It turns out that linear repetitivity (or linear recurrence) implies validity of a subadditive ergodic theorem [5]. Such a subadditive ergodic theorem is useful in application to Schrödinger operators and to lattice gas theory [2, 3, 8-10, 17] (see Sect. 9 of [19] for recent applications to diffraction theory as well). Now, for subshifts, it is possible to characterize validity of a certain subadditive ergodic theorem by positivity of certain weights as shown by one of the authors in [16]. This positivity of weights can be considered as an averaged version of linear repetitivity. This raises two questions:

Q1: Can validity of a subadditive ergodic theorem be characterized for Delone sets in terms of positivity of certain weights?

Q2: What is the relationship between the positivity of weights and linear repetitivity?

Our main results deal with these questions. Theorem 1 shows that positivity of certain weights is indeed equivalent to validity of a subadditive ergodic theorem. This answers Question 1. Theorem 2 characterizes linear repetitivity in terms of positivity of these weights combined with a condition on the shape of return patterns. This gives an answer to Question 2. In the case of symbolic dynamics analogues to Theorem 2 have been obtained earlier. In particular, there is some unpublished work of Monteil [22] characterizing linear repetitivity in terms of positivity of weights and a repulsion property as well as a more general result obtained by Boshernitzan [4]. Boshernitzan's result shows equivalence of linear repetitivity and positivity of weights. Our result and its proof are inspired by [4]. Condition (U) appearing below is not needed in [4] (see Remark 5 at the end of the paper as well).

\section{Notation and Results}

Fix $N \in \mathbb{N}$. We consider subsets of $\mathbb{R}^{N}$. The Euclidean distance on $\mathbb{R}^{N}$ is denoted by $\varrho$. The closed ball around $p \in \mathbb{R}^{N}$ with radius $R$ is denoted by $B_{R}(p)$. We write $B_{R}$ for the closed ball $B_{R}(\mathbf{0})$ (around the origin $\mathbf{0} \in \mathbb{R}^{N}$ ).

A subset $\Lambda \subset \mathbb{R}^{N}$ is called uniformly discrete if its packing radius

$$
r_{\text {pack }}(\Lambda):=\inf \left\{\frac{1}{2} \varrho(x, y): x, y \in \Lambda, x \neq y\right\}
$$

is positive. A subset $\Lambda$ is called relatively dense if its covering radius

$$
r_{\mathrm{cov}}(\Lambda):=\sup \left\{\varrho(p, \Lambda): p \in \mathbb{R}^{N}\right\}
$$

is finite. A set which is both relatively dense and uniformly discrete is called a Delone set. 
Let $\mathbb{R}^{+}=\{r \in \mathbb{R} \mid r>0\}$ stand for the set of positive real numbers.

Given a Delone set $\Lambda$, the Cartesian product

$$
\mathcal{P}_{\Lambda}=\Lambda \times \mathbb{R}^{+}
$$

is referred to as the collection of ball patterns on $\Lambda$. A ball pattern

$$
P=(x, R) \in \Lambda \times \mathbb{R}^{+}=\mathcal{P}_{\Lambda}
$$

is determined by its center $c(P)=x$ and its radius $r(P)=R$. Henceforth we often abbreviate $\mathcal{P}_{\Lambda}$ to just $\mathcal{P}$ when the Delone set $\Lambda$ is clear from context.

Given a ball pattern $P=(x, R)$, its patch is defined as the finite set

$$
\operatorname{patch}(P)=\operatorname{patch}(x, R):=(\Lambda-x) \cap B_{R} \subset \mathbb{R}^{N} .
$$

Clearly, every patch contains the origin $\mathbf{0} \in \mathbb{R}^{N}$.

A subset $A \subset \mathbb{R}^{N}$ is called discrete if $\sharp\left(A \cap B_{R}\right)<\infty$ for all $R>0$. Here $\sharp(A)=$ $\sharp A$ stands for the cardinality of a set $A$.

A Delone set $\Lambda$ is said to be of finite local complexity (FLC) if the difference set $\Lambda-\Lambda=\{x-y: x, y \in \Lambda\}$ is a discrete subset of $\mathbb{R}^{N}$. Note that $\Lambda-\Lambda$ is exactly the union of all patches:

$$
\Lambda-\Lambda=\bigcup_{x \in \Lambda, R \in \mathbb{R}^{+}} \operatorname{patch}(x, R),
$$

and one verifies that (FLC) is equivalent to the following condition:

$$
\left(\mathrm{FLC}_{1}\right) \quad \sharp(\{\operatorname{patch}(x, R) \mid x \in \Lambda\})<\infty \quad \text { for all } R>0 \text {. }
$$

(For every $R>0$, there is only a finite number of patches for ball patterns of radius $R$ ).

In fact, both (FLC) and $\left(\mathrm{FLC}_{1}\right)$ are equivalent to the following condition:

$$
\left(\mathrm{FLC}_{2}\right) \quad \sharp(\{\operatorname{patch}(x, R) \mid x \in \Lambda\})<\infty \quad \text { for } R=2 r_{\mathrm{cov}}(\Lambda)
$$

(see (2.2) for the definition of $r_{\operatorname{cov}}(\Lambda)$ ). For proofs of the equivalence of the above versions of (FLC), we refer to [12]. Given a ball pattern $P=(x, R) \in \mathcal{P}_{\Lambda}$, its locator set $L_{P}$ is defined by the formula

$$
L_{P}(\Lambda)=\{y \in \Lambda: \operatorname{patch}(y, R)=\operatorname{patch}(x, R)\} .
$$

A Delone set $\Lambda$ is said to be repetitive if

$$
r_{\operatorname{cov}}\left(L_{P}\right)<\infty \text { for any } P \in \mathcal{P}_{\Lambda},
$$

i.e., if the locator set $L_{P}$ of any ball pattern $P \in \mathcal{P}_{\Lambda}$ is relatively dense (equivalently, forms a smaller Delone set).

A Delone set $\Lambda$ is called strongly repetitive (compare Definition 2.5 in [14]) if

$$
r_{\mathrm{cov}}\left(L_{P}\right) \leq R(r) \text { for any } P=(x, r) \in \mathcal{P}_{\Lambda},
$$


where the constant $R(r)<\infty$ depends only on the radius $r=r(P)$ of the ball pattern $P$.

Repetitive Delone sets $\Lambda$ do not need to satisfy (FLC). One can prove that a repetitive Delone set satisfies (FLC) if and only if it is strongly repetitive.

In the paper we deal mostly with repetitive Delone sets satisfying (FLC), i.e., strongly repetitive Delone sets.

A Delone set $\Lambda$ is said to be nonperiodic if $\Lambda-x \neq \Lambda$ for all $x \in \mathbb{R}^{N}$ with $x \neq \mathbf{0}$.

Denote by $\mathcal{B}$ the family of bounded subsets in $\mathbb{R}^{N}$. Given a bounded set $Q \in \mathcal{B}$ and a ball pattern $P=(x, R) \in \mathcal{P}_{\Lambda}$, we define the set

$$
L_{P}(Q)=\left\{y \in L_{P}: B_{R}(y) \subset Q\right\}
$$

and two quantities $\sharp_{P} Q, \sharp_{P}^{\prime} Q$ (valued in nonnegative integers) as follows.

The number $\sharp_{P} Q$ (called the number of copies of $P$ in $Q$ ) is defined by the formula

$$
\sharp{ }_{P} Q:=\sharp\left(L_{P}(Q)\right) \text {. }
$$

The number $\sharp_{P}^{\prime} Q \leq \sharp_{P} Q$ (called the maximal number of completely disjoint copies of $P$ in $Q$ ) is defined by the formula

$$
\sharp_{P}^{\prime} Q:=\max \left\{\sharp(A): A \subset L_{P}(Q) \text { such that } \varrho(x, y)>2 R \text { for all } x, y \in A, x \neq y\right\} \text {. }
$$

(Note that the inequality $\varrho(x, y)>2 R$ in the preceding line is equivalent to the requirement that $\left.B_{R}(x) \cap B_{R}(y)=\emptyset\right)$.

We write $|\cdot|$ for the Lebesgue measure in $\mathbb{R}^{N}$. Clearly $\left|B_{R}\right|=R^{N}\left|B_{1}\right|$.

Next, given a ball pattern $P=(x, R) \in \mathcal{P}_{\Lambda}$, we define two quantities, $v(P)$ and $v^{\prime}(P)$, as follows. The lower density of $P, v(P)$, is defined by the formula

$$
v(P):=\liminf _{|C| \rightarrow \infty} \frac{\sharp{ }_{P} C \cdot\left|B_{R}\right|}{|C|}=\left|B_{1}\right| \cdot \liminf _{|C| \rightarrow \infty} \frac{\sharp_{P} C \cdot R^{N}}{|C|},
$$

and the lower reduced density of $P, v^{\prime}(P)$, is defined by the formula

$$
v^{\prime}(P):=\liminf _{|C| \rightarrow \infty} \frac{\sharp_{P}^{\prime} C \cdot\left|B_{R}\right|}{|C|}=\left|B_{1}\right| \cdot \liminf _{|C| \rightarrow \infty} \frac{\sharp_{P}^{\prime} C \cdot R^{N}}{|C|} .
$$

Henceforth, when writing $|C| \rightarrow \infty$, it is always meant that $C$ runs over the cubes in $\mathbb{R}^{N}$.

One easily verifies that, for any Delone set $\Lambda$ and any ball pattern $P \in \mathcal{P}_{\Lambda}$, the inequalities

$$
\begin{aligned}
& 0 \leq v^{\prime}(P) \leq v(P)<\infty, \\
& v^{\prime}(P) \leq\left|B_{1}\right|\left(\frac{\sqrt{N}}{2}\right)^{N}, \quad \text { and } \\
& v^{\prime}(P)>0 \quad \text { (assuming that } \Lambda \text { is repetitive) }
\end{aligned}
$$


hold. (Inequality (2.14) follows from the observation that the diameter of a cube is smaller than $2 R$ if its sidelength is smaller than $\frac{2 R}{\sqrt{N}}$.)

A Delone set $\Lambda$ is said to satisfy positivity of quasiweights (PQ) if

$$
\text { (PQ) } \quad w^{\prime}:=\inf _{\substack{P \in \mathcal{P} \\ r(P) \geq 1}} v^{\prime}(P)>0
$$

(see (2.12)). A Delone set $\Lambda$ is said to satisfy positivity of weights (PW) if

$$
(\mathrm{PW}) \quad w:=\inf _{\substack{P \in \mathcal{P} \\ r(P) \geq 1}} v(P)>0
$$

(see (2.11)). The constant 1 (in (2.16) and (2.17)) is arbitrary; it can be replaced by any other positive constant.

The following is a useful observation.

Remark 1 Let $\Lambda$ be a Delone set. Then $w^{\prime} \leq w$, and the following implications hold:

$(\Lambda$ satisfies $\mathrm{PQ}) \Rightarrow(\Lambda$ satisfies $\mathrm{PW}) \Rightarrow(\Lambda$ is repetitive and has $(\mathrm{FLC}))$.

Indeed, $w^{\prime} \leq w$ follows from (2.13)), and the implications are straightforward.

Recall that $\mathcal{B}$ stands for the family of bounded subsets in $\mathbb{R}^{N}$. A real-valued function $F: \mathcal{B} \rightarrow \mathbb{R}$ is called subadditive if

$$
\text { (subadditivity) } \quad F\left(Q_{1} \cup Q_{2}\right) \leq F\left(Q_{1}\right)+F\left(Q_{2}\right)
$$

whenever $Q_{1}, Q_{2} \in \mathcal{B}$ are disjoint. Such a function $F$ is said to be $\Lambda$-invariant if

$$
F(Q)=F(t+Q) \quad \text { whenever } t+(Q \cap \Lambda)=(t+Q) \cap \Lambda .
$$

A Delone set $\Lambda$ is said to satisfy a subadditive ergodic theorem (SET) if for any subadditive invariant function $F$, the limit

$$
\text { (SET) } \mu(F)=\lim _{|C| \rightarrow \infty} \frac{F(C)}{|C|}
$$

exists. (Recall that, under our conventions, $C$ runs over the cubes in $\mathbb{R}^{N}$ ).

Theorem 1 Let $\Lambda$ be a repetitive Delone set with $(F L C)$. Then (SET) is equivalent to (PQ).

Remark 2 It is possible to replace the cubes appearing in the limit of (SET) by cube like sequences. We refrain from giving details.

The theorem has the following corollary.

Corollary 1 If a Delone set $\Lambda$ satisfies $(P Q)$, then for every ball pattern $P \in \mathcal{P}_{\Lambda}$, the frequency $\lim _{|C| \rightarrow \infty} \frac{\sharp P C}{|C|}$ exists. 
Proof of Corollary 1 By Theorem 1, $\Lambda$ satisfies (SET). The claim of Corollary 1 follows because $F(Q)=-\sharp_{P}(Q)$ is a subadditive function on $\mathcal{B}$.

Remark 3 It is known that the dynamical system associated to a Delone set $\Lambda$ of finite local complexity satisfying (SET) is uniquely ergodic $[15,21]$. In fact, unique ergodicity is equivalent to existence of pattern frequencies. Thus, Corollary 1 shows that (PQ) is also a sufficient condition for unique ergodicity of this system.

We will be concerned with further combinatorial quantities associated with Delone sets $\Lambda$. These quantities will be discussed next.

A Delone set $\Lambda$ is called linearly repetitive (LR) if

$$
\text { (LR) } \sup _{\substack{P \in \mathcal{P}_{\Lambda} \\ r(P) \geq 1}} \frac{r_{\mathrm{cov}}\left(L_{P}^{\Lambda}\right)}{r(P)}<\infty .
$$

It is clear that linearly repetitive Delone sets are strongly repetitive.

A Delone set $\Lambda$ is said to satisfy the repulsion property (RP) if

$$
\text { (RP) } \inf _{P \in \mathcal{P}_{\Lambda}} \frac{r_{\text {pack }}\left(L_{P}^{\Lambda}\right)}{r(P)}>0 \text {. }
$$

We finally need the notion of return pattern. For subshifts, an intense study of this notion has been carried out in work of Durand [6, 7]. The analogue for Delone sets (or rather tilings) has then been investigated by Priebe [25] (see, e. g., [20, 26] as well).

Recall that a subset $\Gamma \subset \mathbb{R}^{N}$ is called discrete if $\sharp\left(\Gamma \cap B_{R}\right)<\infty$ for all $R>0$.

Given a discrete subset $\Gamma \subset \mathbb{R}^{N}$ of cardinality $\sharp(\Gamma) \geq 2$, the Voronoi cell of some $x \in \Gamma$ is defined by the formula

$$
V_{x}(\Gamma):=\left\{p \in \mathbb{R}^{N}: \varrho(p, x) \leq \varrho(p, y) \text { for all } y \in \Gamma\right\} .
$$

(Recall that $\varrho(\cdot, \cdot)$ stands for the Euclidean distance in $\mathbb{R}^{N}$ ). The collection

$$
\widetilde{V}(\Gamma)=\left\{V_{x}(\Gamma) \mid x \in \Gamma\right\}
$$

is called the Voronoi partition of $\mathbb{R}^{N}$ corresponding to $\Gamma$.

Each Voronoi cell $V_{x}=V_{x}(\Gamma)$ (an element of this partition) is a convex subset in $\mathbb{R}^{N}$ with nonempty interior containing $x$.

The distortion $\lambda\left(V_{x}\right)$ of the Voronoi cell $V_{x}$ is defined as the ratio

$$
\lambda\left(V_{x}\right)=\frac{r_{\text {out }}\left(V_{x}\right)}{r_{\text {in }}\left(V_{x}\right)} \in[1, \infty]
$$

of the outer and inner radii of the cell $V_{x}$ (denoted $r_{\text {out }}\left(V_{x}\right)$ and $r_{\text {in }}\left(V_{x}\right)$, respectively). These are defined as follows:

$$
r_{\text {in }}\left(V_{x}\right):=\sup \left\{R>0: B_{R}(x) \subset V_{x}\right\}=\inf _{y \in \partial V_{x}} \varrho(x, y)=\varrho\left(x, \partial V_{x}\right),
$$




$$
r_{\text {out }}\left(V_{x}\right):=\inf \left\{R>0: V_{x} \subset B_{R}(x)\right\}=\sup _{y \in \partial V_{x}} \varrho(x, y) .
$$

If $\Lambda$ is a Delone set, the Voronoi cells $V_{x}(\Lambda) \in \widetilde{V}(\Lambda)$ are easily seen to form compact polytopes in $\mathbb{R}^{N}$, and their distortions are uniformly bounded by $\frac{r_{\operatorname{cov}}(\Lambda)}{r_{\operatorname{pack}}(\Lambda)}$.

By a distortion of a Delone set $\Lambda$ we mean the supremum of distortions of its Voronoi cells: $\lambda(\Lambda)=\sup _{x \in \Lambda} \lambda\left(V_{x}\right)$.

It follows that the distortion of any Delone set $\Lambda$ must be finite:

$$
1<\lambda(\Lambda) \leq \frac{r_{\mathrm{cov}}(\Lambda)}{r_{\mathrm{pack}}(\Lambda)}<\infty .
$$

Assume from now on that $\Lambda$ is a fixed repetitive Delone set. Then for every ball pattern $P \in \mathcal{P}_{\Lambda}$, the locator set $L_{P}=L_{P}^{\Lambda}$ (see (2.1)) is itself a Delone set which determines its own Voronoi partition

$$
\widetilde{V}_{P}=\widetilde{V}\left(L_{P}\right)=\left\{V_{x}\left(L_{P}\right) \mid x \in L_{P}\right\} .
$$

A Delone set $\Lambda$ is said to satisfy uniformity of return words (U) if the distortions of all its locator sets are uniformly bounded, i.e., if

$$
\text { (U) } \sup _{P \in \mathcal{P}_{\Lambda}} \lambda\left(L_{P}\right)<\infty \text {. }
$$

Theorem 2 Let $\Lambda$ be a nonperiodic Delone set. Then the following assertions are equivalent:

(i) The set $\Lambda$ satisfies (LR).

(ii) The set $\Lambda$ satisfies (PQ) and (U).

(iii) The set $\Lambda$ satisfies (PW) and (U).

For definitions of the properties (LR), (PQ), (PW) and (U) of a Delone set $\Lambda$ see (2.21), (2.16), (2.17) and (2.24), respectively.

Remark 4 Note that a Delone set satisfying (LR) or (PQ) or (PW) must be repetitive and satisfy (FLC) as discussed above.

In Sect. 3, we provide a proof for Theorem 1. Section 4 deals with Theorem 2.

\section{A Subadditive Ergodic Theorem}

Proof of Theorem 1 We have to show that conditions (SET) and (PQ) are equivalent. The analogue result in the setting of symbolic dynamics is given in [16]. Here, we indicate the necessary modifications.

$(\mathrm{PQ}) \Rightarrow(\mathrm{SET})$ : The proof given in [16] is easily carried over to show that (PQ) implies existence of the $\operatorname{limit}_{|C| \rightarrow \infty} \frac{F(C)}{|C|}$ where $C$ runs over the cubes in $\mathbb{R}^{N}$. A similar reasoning can also be found in the proof of (LR) $\Rightarrow$ (SET) given in [5].

$(\mathrm{SET}) \Rightarrow(\mathrm{PQ})$ : Assume to the contrary that (SET) holds but (PQ) fails. 
Since (PQ) does not hold, there exists a sequence $\left(P_{n}\right)$ of ball patterns with $v^{\prime}\left(P_{n}\right) \rightarrow 0$ (see (2.12)). By repetitivity, $v^{\prime}\left(P_{n}\right)>0$ for all $n$ (see (2.15)). As $\Lambda$ satisfies (FLC), this implies $r\left(P_{n}\right) \rightarrow \infty$.

With notation as in Sect. 2, define the real-valued functions $F_{n}: \mathcal{B} \rightarrow \mathbb{R}$ by

$$
F_{n}(Q):=\sharp_{P_{n}}^{\prime}(Q)\left|B_{r\left(P_{n}\right)}\right| \quad(n \geq 1) .
$$

(Recall that $\mathcal{B}$ stands for the family of all bounded subsets of $\mathbb{R}^{n}$, and $\sharp_{P_{n}}^{\prime}(Q)$ is defined as in (2.10)).

Observe that each function $-F_{n}$ is subadditive and also $\Lambda$-invariant. By (SET), the limits $\lim _{|C| \rightarrow \infty} \frac{F_{n}(C)}{|C|}$ exist and are equal to $v\left(P_{n}\right)=\mu(F)$ (see (2.20)).

Since $v\left(P_{n}\right) \rightarrow 0$, it follows that

$$
v\left(P_{n}\right)=\mu\left(F_{n}\right)=\lim _{|C| \rightarrow \infty} \frac{F_{n}(C)}{|C|} \rightarrow 0 \quad(n \rightarrow \infty) .
$$

Select now a positive $\varepsilon<\frac{\left|B_{1}\right|}{2^{N}}$. Using the relations $v\left(P_{n}\right) \rightarrow 0$ and $r\left(P_{n}\right) \rightarrow \infty$, one constructs inductively a subsequence $\left(P_{n_{k}}\right)$ of $\left(P_{n}\right)$ such that

$$
\frac{1}{|C|} \sum_{j=1}^{k} F_{n_{j}}(C)<\varepsilon \quad \text { and } \quad r\left(P_{n_{k+1}}\right)>r\left(P_{n_{k}}\right)
$$

for all $k \geq 1$ and all cubes $C$ with sidelengths $s(C) \geq \frac{1}{2} r\left(P_{n_{k+1}}\right)$.

Define the function $F: \mathcal{B} \rightarrow \mathbb{R}$ by $F(Q):=\sum_{j=1}^{\infty} F_{n_{j}}(Q)$.

Since $r\left(P_{n}\right) \rightarrow \infty$, only finitely many terms in this sum do not vanish. As each function $-F_{n}$ is subadditive and $\Lambda$-invariant, so is $-F$. Therefore, (SET) implies existence of the limit $\mu(F)=\lim _{|C| \rightarrow \infty} \frac{F(C)}{|C|}$.

For any $k \geq 1$ and for any cube $C$ with its sidelength $s(C)$ satisfying the inequalities $\frac{1}{2} r\left(P_{n_{k+1}}\right) \leq s(C)<2 r\left(P_{n_{k+1}}\right)$, we obtain

$$
\frac{F(C)}{|C|}=\sum_{j=1}^{\infty} \frac{F_{n_{j}}(C)}{|C|}=\sum_{j=1}^{k} \frac{F_{n_{j}}(C)}{|C|}<\varepsilon .
$$

On another hand, for a cube $C$ with sidelength $2 r\left(P_{n_{k}}\right)$ and center of mass in $x \in L_{P_{n_{k}}}$, we have

$$
\frac{F(C)}{|C|} \geq \frac{F_{n_{k}}(C)}{|C|} \geq \frac{\left|B_{r\left(P_{n_{k}}\right)}\right|}{|C|}=\frac{\left|r\left(P_{n_{k}}\right)\right|^{N} \cdot\left|B_{1}\right|}{\left|2 r\left(P_{n_{k}}\right)\right|^{N}}=\frac{\left|B_{1}\right|}{2^{N}} .
$$

It follows that $\frac{\left|B_{1}\right|}{2^{N}} \leq \mu(F) \leq \varepsilon$, contrary to the choice of $\varepsilon$.

This completes the proof of Theorem 1 .

\section{Linear Repetitive Delone Sets}

In this section Theorem 2 is proved. We need several auxiliary results. 
Proposition 4.1 Let $\Lambda$ be a nonperiodic Delone set. Then (LR) implies (RP).

For the definitions of properties (LR) and (RP), see (2.21) and (2.22).

Proof For linearly repetitive nonperiodic tilings, this (and in fact a slightly stronger result) is proven in Lemma 2.4 of [29]. That proof carries easily over to Delone sets, as discussed in Lemma 2.1 in [18] (see [14] and [7] as well).

Proposition 4.2 Let $\Lambda$ be a nonperiodic Delone set. Then, (LR) implies (U).

Proof By Proposition 4.1, $\Lambda$ satisfies (RP). Now, from (LR) and (RP) there follows existence of positive constants $c$ and $C$ depending only on $\Lambda$ such that the inequalities $r_{\text {pack }}\left(L_{P}\right) \geq \operatorname{cr}(P)$ and $r_{\text {cov }}\left(L_{P}\right)<C r(P)$ hold for all ball patterns $P$. These imply that $\frac{r_{\text {cov }}\left(L_{P}\right)}{r_{\text {pack }}\left(L_{P}\right)}<\frac{C}{c}$. For $x \in L_{P}$, we have the following inclusions for the set $V_{x}\left(L_{P}\right)$ (defined in (2.23)):

$$
B\left(x, r_{\mathrm{pack}}\left(L_{P}\right)\right) \subset V_{x}\left(L_{P}\right) \subset B\left(x, r_{\mathrm{cov}}\left(L_{P}\right)\right),
$$

see Corollary 5.2 in [28] for details. The estimate

$$
\lambda\left(V_{x}(P)\right)=\frac{r_{\text {out }}\left(V_{x}(P)\right)}{r_{\text {in }}\left(V_{x}(P)\right)} \leq \frac{r_{\mathrm{cov}}\left(L_{P}\right)}{r_{\text {pack }}\left(L_{P}\right)} \leq \frac{C r(P)}{c r(P)}<\frac{C}{c}
$$

implies $\lambda\left(L_{P}\right) \leq \frac{C}{c}$, which completes validation of $(\mathrm{U})$ (see (2.24)).

Proposition 4.3 Let $\Lambda$ be a Delone set. Then (LR) implies (PQ).

Proof By definition, (LR) implies that every pattern $P=(x, R) \in \mathcal{P}$ occurs in every box of sidelength $C R$. We can now partition any sufficiently large cube into smaller cubes of sidelength $3 C R$ up to its boundary. Each of these smaller cubes contains a cube of sidelength $C R$ "in the middle," i. e., with distance $C R$ to the boundary. Choosing a copy of $P$ in each of these middle cubes, we easily obtain the statement.

We will need two further lemmas before we can give the proof of the second main theorem.

Lemma 4.4 Let $m>n \geq 1$ be integer numbers. Then $\sum_{k=n}^{m} \frac{1}{k} \geq \ln \left(\frac{m+1}{n}\right)$.

Proof As the function $f(x)=\frac{1}{x}$ is decreasing on the interval $] 0,+\infty[$, we find $\sum_{k=n}^{m} \frac{1}{k} \geq \int_{n}^{m+1} \frac{d x}{x}=\ln \left(\frac{m+1}{n}\right)$.

Lemma 4.5 Let $\Lambda$ be a Delone set satisfying (PW) and (U), and let

$$
P=\left(x_{0}, R\right) \in \Lambda \times \mathbb{R}^{+}=\mathcal{P}_{\Lambda}=\mathcal{P}
$$


be a ball pattern of radius $R:=r(P) \geq 3$. Then, for all points $x \in L_{P}$ (see (2.8)), the following inequality holds:

$$
r_{\text {in }}\left(V_{x}\left(L_{P}\right)\right) \leq c R
$$

where $c=\max \left(2,4 \exp \left(\frac{6^{N}}{2 w N}\right)\right)$, and the constant $w$ is coming from the definition of (PW).

Proof Set $d$ to be the distance from $x$ to the closest point in the set $L_{P}^{\Lambda} \backslash\{x\}$ :

$$
d:=\min _{\substack{y \in L_{P}^{\Lambda} \\ y \neq x}} \varrho(x, y) .
$$

We may assume that $d>4 R$ because otherwise inequality in (4.1) holds with $c=2$.

For $t \in \mathbb{R}$, let $E(t)$ denote the greatest integer smaller than $t$. Set

$$
\begin{array}{ll}
d^{\prime}=E\left(\frac{d}{3}\right), & R^{\prime}=E(R), \\
m=d^{\prime}-R^{\prime}, & R_{k}=R^{\prime}+k \quad(k \geq 0) .
\end{array}
$$

Since $\frac{d}{3}-R>\frac{R}{3} \geq 1$, it follows that $m \geq 1$, and we have

$$
2<3 \leq R^{\prime}=R_{0} \leq R<R_{1}<\cdots<R_{m}=d^{\prime} \leq \frac{d}{3} .
$$

Consider the following $m$ ball patterns:

$$
P_{k}=\left(x, 3 R_{k}\right) \in \mathcal{P}, \quad 1 \leq k \leq m .
$$

We list the following observations (Facts 1-5):

Fact 1 The following inclusions are fulfilled:

$$
\left.x \in L_{P_{i}} \subset L_{P_{j}} \subset L_{P} \quad \text { (for } 1 \leq j \leq i \leq m\right) .
$$

Fact 2 For $z \in L_{P_{i}}$ with $1 \leq i \leq k$, we have $L_{P} \cap B_{2 R_{i}}(z)=\{z\}$.

In fact even more is true:

Fact 3 For $z \in L_{P_{i}}$ with $1 \leq i \leq k$, we have

$$
\left(L_{P}-z\right) \cap B_{2 R_{i}} \stackrel{1}{=}\left(L_{P}-x\right) \cap B_{2 R_{i}} \stackrel{2}{=}\{0\} .
$$

Indeed, the first equality $\stackrel{1}{=}$ holds since $R=r(P) \leq R_{i}$ and both $x, z \in L_{P_{i}}$. The second equality $\stackrel{2}{=}$ follows from the inequalities $2 R_{i}<3 R_{i} \leq 3 R_{m}<d$. 
Fact 4 If $z \in L_{P_{i}}$ and $y \in L_{P_{j}}$ with $z \neq y$ and $i, j \in\{1,2, \ldots, m\}$, then

$$
\left(B_{R_{i}}(z) \backslash B_{R_{i-1}}(z)\right) \cap\left(\left(B_{R_{j}}(y) \backslash B_{R_{j-1}}(y)\right)\right)=\emptyset .
$$

Indeed, assuming (without loss of generality) that $i \geq j$, we have $y \in L_{P_{j}} \subset L_{P}$ (Fact 1), and, in view of the Fact 2, we obtain $\varrho(x, y)>2 R_{i}$, whence the claim of Fact 4 follows. The next observation (Fact 5) is obvious.

Fact 5 If $i \neq j$ and $z \in L_{P_{i}} \cap L_{P_{j}}$ with $i, j \in\{1,2, \ldots, m\}$, then Eq. (4.2) takes place.

Given the disjointness observations (4.2) (Facts 4 and 5), the inequality

$$
\sum_{k=1}^{m} \sharp P_{k}\left(\Phi_{d^{\prime}}(C)\right)\left|B_{R_{k}} \backslash B_{R_{k-1}}\right| \leq|C|
$$

holds for any cube $C$. Here, for $Q \subset \mathbb{R}^{N}$ and $s>0$, we denote by $\Phi_{s}(Q)$ the set of points of $Q$ with distance at least $s$ to the boundary of $Q$.

Let $C_{n}$ be an arbitrary sequence of cubes with $\left|C_{n}\right| \rightarrow \infty$. Then

$$
1 \geq \sum_{k=1}^{m} \frac{\sharp P_{k}\left(\Phi_{d^{\prime}}\left(C_{n}\right)\right)}{\left|C_{n}\right|}\left|B_{R_{k}} \backslash B_{R_{k-1}}\right| \text { for all } n \text {. }
$$

By letting $n \rightarrow \infty$ we obtain from (PW) (see (2.17) for the definition of (PW))

$$
1 \geq \sum_{k=1}^{m} \frac{w}{\left|P_{k}\right|}\left|B_{R_{k}} \backslash B_{R_{k-1}}\right| \geq \frac{w}{3^{N}} \cdot \sum_{s=R^{\prime}+1}^{d^{\prime}} \frac{s^{N}-(s-1)^{N}}{s^{N}} .
$$

Using the inequalities $\frac{s^{N}-(s-1)^{N}}{s^{N}}>\frac{N(s-1)^{N-1}}{s^{N}}>\frac{N(s / 2)^{N-1}}{s^{N}}=\frac{N}{s \cdot 2^{N-1}}$ with $s \geq 2$ and then Lemma 4.4, we can rewrite (4.3) in the form

$$
1 \geq \frac{w}{3^{N}} \cdot \frac{N}{2^{N-1}} \cdot \sum_{s=R^{\prime}+1}^{d^{\prime}} \frac{1}{s} \geq \frac{2 w N}{6^{N}} \cdot \ln \left(\frac{d^{\prime}+1}{R^{\prime}+1}\right) .
$$

It follows that $\frac{d}{3} \leq d^{\prime}+1 \leq\left(R^{\prime}+1\right) \cdot \exp \left(\frac{6^{N}}{2 w N}\right)$. In view of the inequalities $R^{\prime}+1<$ $R+1 \leq \frac{4 R}{3}$ (which hold for $R \geq 3$ ), we obtain

$$
d<4 R \exp \left(\frac{6^{N}}{2 w N}\right)
$$

completing the proof of Lemma 4.5.

We can now turn to the following:

Proof of Theorem 2 Through the present proof, $\Lambda$ is assumed to be a nonperiodic Delone set, and the properties (PQ), (U), (LR), and (FLC) refer to it. 
We show $(\mathrm{LR}) \Longrightarrow((\mathrm{PQ})$ and $(\mathrm{U})) \Longrightarrow((\mathrm{PW})$ and $(\mathrm{U})) \Longrightarrow(\mathrm{LR})$.

The implication $(\mathrm{LR}) \Longrightarrow((\mathrm{PQ})$ and $(\mathrm{U}))$ immediately follows from Propositions 4.2 and 4.3.

The implication $((\mathrm{PQ})$ and $(\mathrm{U})) \Longrightarrow((\mathrm{PW})$ and $(\mathrm{U}))$ is clear in view of (2.18).

In what follows, we establish linear repetitivity (LR) of a nonperiodic Delone set $\Lambda$ satisfying (PW) and (U). (Note that $\Lambda$ is repetitive and also satisfies (FLC) (both properties follow from (PW))).

Let $t \in \mathbb{R}^{N}$ be arbitrary, and let $P=\left(x_{0}, R\right) \in \mathcal{P}$ be a ball pattern in $\Lambda$ with $R:=r(P) \geq 3$. Select $x \in L_{P}$ such that $t \in V_{x}$. By Lemma 4.5 we have

$$
r_{\text {in }}\left(V_{x}\right) \leq c R
$$

and then, by $(U)$ (see (2.24)),

$$
r_{\text {out }}\left(V_{x}\right) \leq c \sigma R
$$

As the ball of radius $r_{\text {out }}\left(V_{x}\right)$ around $t$ contains $x$, we obtain

$$
r_{\text {cov }}\left(L_{P}\right) \leq c \sigma R \quad \text { whenever } R \geq 3 \text {. }
$$

Since the collection of patches corresponding to ball patterns of radius $\leq 3$ is finite in view of (FLC) of $\Lambda$ (see (2.6)), the validity of (LR) follows from (4.4) (for the definition of (LR) see (2.21)). This completes the proof of Theorem 2.

Remark 5 Let us shortly comment on assumption (U) appearing above. It can be understood as a form of isotropy condition. From this point of view it seems reasonable that it is not necessary in the one-dimensional case. Indeed, in this case one does not need to work with the "centers" of the Voronoi cells but can rather work with intervals to the right (or left) of the points [4]. One might wonder whether assumption (U) be dropped in the higher-dimensional situation as well. On the other hand, it seems also an interesting question which restrictions are imposed on the geometry of a discrete set by condition $(\mathrm{U})$ alone.

Acknowledgements M. Boshernitzan was supported in part by NSF Grant: DMS-1102298.

This work was partially supported by the German Science Foundation (DFG). Part of this work was done while one of the authors (D. Lenz) was visiting Rice University. He would like to thank the Department of Mathematics for hospitality.

\section{References}

1. Baake, M., Moody (eds), R.V.: Directions in Mathematical Quasicrystals. CRM Monogr. Ser., vol. 13. Am. Math. Soc., Providence (2000)

2. Besbes, A.: Uniform subadditive ergodic theorem on aperiodic linearly repetitive tilings and applications. Rev. Math. Phys. 20, 597-623 (2008)

3. Besbes, A.: Contribution à l'étude de quelques systèmes quasi-cristallographiques. Ph.D. Thesis, Paris (2008)

4. Boshernitzan, M.: Private communication (2012, in preparation)

5. Damanik, D., Lenz, D.: Linear repetitivity. I. Uniform subadditive ergodic theorems and applications. Discrete Comput. Geom. 26, 411-428 (2001) 
6. Durand, F.: A characterization of substitutive sequences using return words. Discrete Math. 179, 89101 (1998)

7. Durand, F.: Linearly recurrent subshifts have a finite number of non-periodic subshift factors. Ergod. Theory Dyn. Syst. 20, 1061-1078 (2000). Erratum: Ergod. Theory Dyn. Syst. 23, 663-669 (2003)

8. Geerse, C.P., Hof, A.: Lattice gas models on self-similar aperiodic tilings. Rev. Math. Phys. 3, 163$221(1991)$

9. Hof, A.: Some remarks on discrete aperiodic Schrödinger operators. J. Stat. Phys. 72, 1353-1374 (1993)

10. Hof, A.: A remark on Schrödinger operators on aperiodic tilings. J. Stat. Phys. 81, 851-855 (1996)

11. Ishimasa, T., Nissen, H.U., Fukano, Y.: New ordered state between crystalline and amorphous in NiCr particles. Phys. Rev. Lett. 55, 511-513 (1985)

12. Lagarias, J.C.: Geometric models for quasicrystals. I. Delone sets of finite type. Discrete Comput. Geom. 21, 161-191 (1999)

13. Lagarias, J.C., Pleasants, P.A.B.: Repetitive delone sets and quasicrystals. Ergod. Theory Dyn. Syst. 23, 831-867 (2003)

14. Lagarias, J.C., Pleasants, P.A.B.: Local complexity of Delone sets and crystallinity. Can. Math. Bull. 45, 634-652 (2002)

15. Lee, J.-Y., Moody, R.V., Solomyak, B.: Consequences of pure point diffraction spectra for multiset substitution systems. Discrete Comput. Geom. 29, 525-560 (2003)

16. Lenz, D.: Uniform ergodic theorems on subshifts over a finite alphabet. Ergod. Theory Dyn. Syst. 22, 245-255 (2002)

17. Lenz, D.: Singular spectrum of Lebesgue measure zero for one-dimensional quasicrystals. Commun. Math. Phys. 227, 119-130 (2002)

18. Lenz, D.: Aperiodic linearly repetitive delone sets are densely repetitive. Discrete Comput. Geom. 31, 323-326 (2004)

19. Lenz, D.: Continuity of eigenfunctions of uniquely ergodic dynamical systems and intensity of Bragg peaks. Commun. Math. Phys. 287, 225-258 (2009)

20. Lenz, D., Stollmann, P.: An ergodic theorem for delone dynamical systems and existence of the integrated density of states. J. Anal. Math. 97, 1-23 (2006)

21. Lenz, D., Stollmann, P.: Delone dynamical systems and associated random operators. In: Operator Algebras and Mathematical Physics (Constanta 2001). Theta Foundation, Bucharest (2003)

22. Monteil, T.: Private communication (2003)

23. Moody, R.V. (ed.) The mathematics of long-range aperiodic order, Waterloo, ON, 1995. NATO Adv. Sci. Inst. Ser. C Math. Phys. Sci., pp. 403- 441 (1997)

24. Patera, J. (ed.): Quasicrystals and Discrete Geometry, Toronto, ON, 1995. Fields Inst. Monogr., vol. 10. Am. Math. Soc., Providence (1998)

25. Priebe, N.: Towards a characterization of self-similar tilings in terms of derived Voronoi tesselations. Geom. Dedic. 79, 239-265 (2000)

26. Priebe-Frank, N., Solomyak, B.: Characterization of planar pseudo-self-similar tilings. Discrete Comput. Geom. 26, 289-306 (2001)

27. Shechtman, D., Blech, I., Gratias, D., Cahn, J.W.: Metallic phase with long-range orientational order and no translational symmetry. Phys. Rev. Lett. 53, 1951-1953 (1984)

28. Senechal, M.: Quasicrystals and Geometry. Cambridge University Press, Cambridge (1995)

29. Solomyak, B.: Nonperiodicity implies unique composition for self-similar translationally finite tilings. Discrete Comput. Geom. 20, 265-279 (1998) 